\title{
An Improved Repetitive Control for Circulating Current Restraining in MMC-MTDC
}

\author{
Yahui Wang*, Yijia Cao, Yong Li, Chang Li \\ College of Electrical and Information Engineering, Hunan University, Changsha 410082, China, 0731- \\ 88822213/0731-88822224 \\ *Corresponding author, e-mail: wangyahui_hnu2@163.com
}

\begin{abstract}
The modular multilevel converter (MMC) is widely used in many important application fields such as high voltage $D C$ transmission system. And the multi-terminal architecture of it attracts many attentions. However, the circulating current of MMC is an inherent problem which is mainly caused by the voltage mismatch between arms and DC bus. In this paper, an advanced repetitive control method is proposed. This method is based on the even-harmonic characteristic of the circulating current and the potential feature of repetitive control that it has an internal integration part. The pole diagram of the closed loop transform function of the proposed control system proves the stability of the proposed method. And according to the simulation results of a three-terminal MMC-MTDC model in PSCAD/EMTDC, the improved repetitive control presents better circulation repression ability and superior anti-interference capability by comparing with traditional PI control method. Additionally, the simulation results also indicate that the proposed repetitive controller can restrain the fluctuation of SM voltage more effectively than PI control.
\end{abstract}

Keywords: Modular multilevel converter (MMC), circulating current, multi-terminal direct current system (MTDC), repetitive control

Copyright (c) 2017 Universitas Ahmad Dahlan. All rights reserved.

\section{Introduction}

With the advantages of large transmission capacity and control flexibility, the power transmission among multiple isolated points can be realized by multi-terminal direct current system (MTDC) which uses voltage source converter (VSC). In this way, VSC-MTDC has significant practical value because it can be applied in many important fields, particularly offshore wind power system [1,2]. MMC is an attractive converter topology of VSC which consists of many sub-modules (SM). Comparing with traditional two-level topology and threelevel topology of VSC, MMC is superior because it has flexible expandability, lower switching frequency, higher power quality and better fault-handling ability due to its redundancy [3-5]. However, MMC has a well-known defect, that is, the circulating current which is caused by the energy imbalance among three phases. This defect may lead to distortion of arm current, enlargement of rated switching capacity, increase of device dissipation and even devices burnout [6-8]. Therefore, the method to restrain circulation current in MMC is necessary to be studied.

The existing methods for circulating current restraining in MMC can be generally divided in two types, that is, hardware methods and software methods. As for the first type, the core idea is to achieve circulating current suppressing effect by changing the hardware parameters. However, hardware methods can only decrease circulating current but cannot eliminate it, and they will increase the volume and cost of the converter and has bad influence on its dynamic performance. For example, reference [9] presents a method to suppress circulating current by appropriately selecting arm inductance. Contrary to hardware methods, software methods are implemented by improving control design based on various principles without changing hardware parameters. The basic principles applied in this area include PI control used in negative sequence double frequency dq rotating coordinate system $[6,7]$ and PR control with the ability to follow a specific frequency harmonic [8]. But they both need large calculation and cannot effectively suppress multiple frequency harmonics at the same time. Accordingly, the repetitive control is proposed to suppress multiple frequency harmonics in MMC. But this control 
strategy has an unavoidable one fundamental period delay due to its internal model control principle. Then, the idea of plug-in repetitive controller is appeared in [10-13], which combines $\mathrm{PI}$ controller with repetitive controller to eliminate not only even harmonics but also odd harmonics. But the steady-state analysis in [14] shows that the circulating current harmonics in MMC are dominated by even harmonics. So reference [15] presents an idea of even-harmonic repetitive control. It can achieve nearly the same harmonic elimination performance as the plugin repetitive control mentioned above. Furthermore, even-harmonic repetitive control needs less memory occupation and has faster dynamic response. But in all the papers mentioned above, the PI controller is as a whole to be paralleled or cascaded with repetitive controller. Though PI controller can improve the dynamic performance of the control system, but simple superposition of two controller will lead to conflict between them. Additionally, in recent year, the circulating current are mostly studied within MMC converter, but it is also worthwhile to be analyzed on the system level in unbalanced grid conditions [16, 17].

In order to avoid the problems mentioned above and keep all the advantages, in this paper, a new circulating current restraining method of MMC is proposed. This method is based on an even-harmonic repetitive controller and a parallel proportional controller. The repetitive controller is proved to have an equivalent internal integration part. And this internal integration part is integrated with the outside proportional controller, so they can realize the function of a PI controller together. By comparing with previous plug-in repetitive control, the proposed method has briefer control structure, faster respond speed and similar harmonic elimination ability. The validity of this method is proved by simulation results of three-terminal MMC system in PSCAD/EMTDC.

This paper is organized as follows. In Section 1, the structure and operating principle of MMC-MTDC is analyzed, and the mechanism of circulating current is also studied. Section 2 presents the principle and design of proposed circulating current restraining method by comparing with traditional repetitive control. Sections 3 validates the effectiveness of proposed control scheme by simulation results. Section 4 concludes the paper.

\section{Structure and Operating Principle of MMC-MTDC \\ 2.1. Structure of MMC-MTDC}

MTDC system contains three or more VSCs, and those converters are connected in series or in parallel or both. In this paper, a parallel three-terminal MMC-MTDC system is studied. In parallel pattern, the DC voltage of each converter is the same, so the power allocation among converters is realized by changing DC current. In Figure 1, the terminal 1 and 2 are sending ends and terminal 3 is a receiving end.

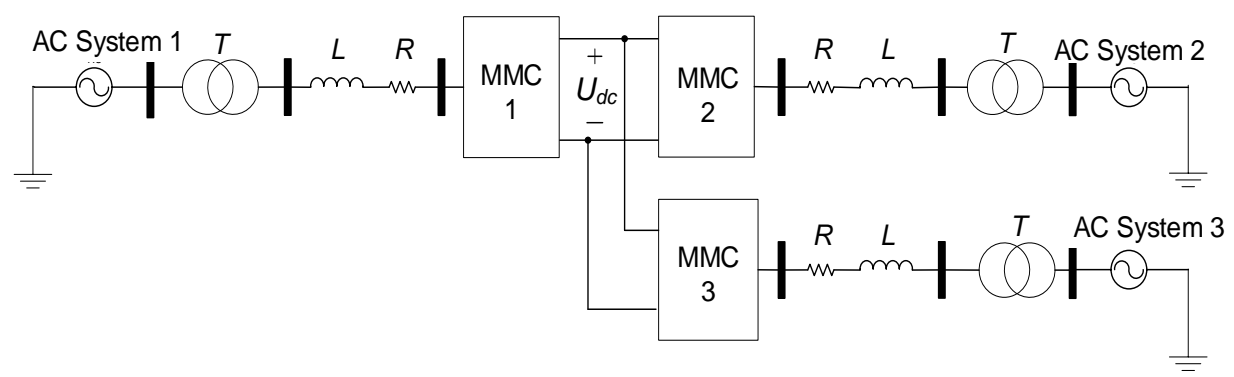

Figure 1. Structure of a three-terminal MMC-MTDC system

Figure 2 shows the structure of a three-phase MMC. This converter has upper and lower arms in each phase, and every arm consists of several cascaded Sub-modules and an arm reactor. And Figure 2 also shows the structure of a Sub-module, it is composed of a halfbridge unit and a capacitor. 


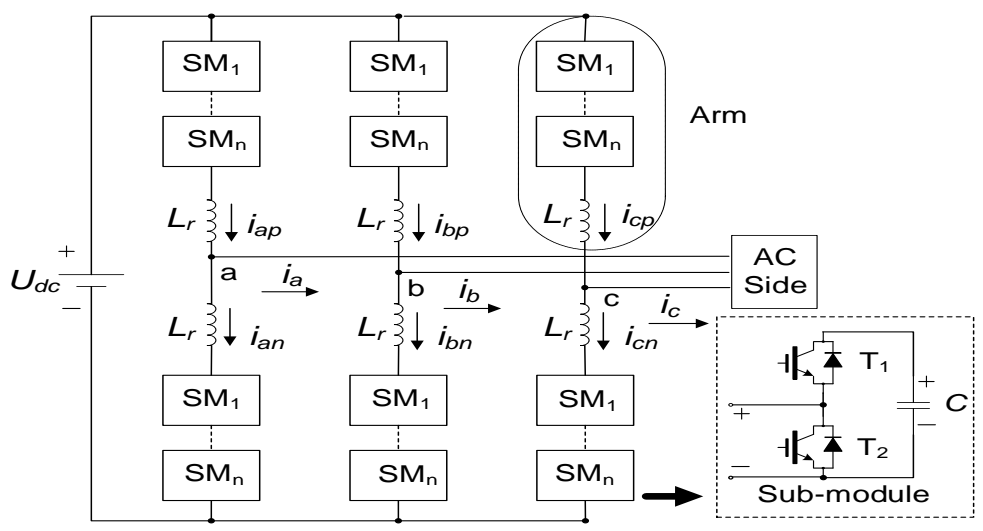

Figure 2. Structure of a three-phase MMC

\subsection{Operating Principle of MMC}

According to Figure 2, the output voltage of a SM can be switched between the capacitor voltage $u_{c}$ and 0 by turning on $T_{1}$ or $T_{2}$. If $s$ is defined as a parameter of switching state function, it equals to 1 when $T_{1}$ is on and $T_{2}$ is off, otherwise $s$ is 0 [18]. So the voltage of upper arm and that of lower arm in $x$ phase can be expressed as formula (1) and (2).

$$
\begin{aligned}
& u_{x p}=\sum_{k-1}^{n}\left(s_{x k p} u_{c x k p}^{0}\right)-\frac{1}{C} s_{x k p} \int_{0}^{t} i_{x p} d t \\
& u_{x n}=\sum_{k-1}^{n}\left(s_{x k n} u_{c x k n}^{0}\right)-\frac{1}{C} s_{x k n} \int_{0}^{t} i_{x n} d t
\end{aligned}
$$

Where $s_{x k p}(k=0 \ldots n$ and $x=a, b, c)$ represents the switching state of the $k$ th SM in upper arm of phase $x$. For example, $s_{a k p}=1$ stands for the $k$ th $S M$ in upper arm of phase a is on, and $s_{a k p}=0$ means it is off. Similarly, $s_{x k n}(k=0 \ldots n$ and $x=a, b, c)$ represents the switching state of the $k$ th SM in lower arm of phase $x$.

To keep DC voltage stable and obtain high-quality sinusoidal AC voltage, on the one hand, the total output voltage of SMs in both upper and lower arms in one phase must equal to $U_{d c}$. On the other hand, the value of $u_{x p}$ and $u_{x p}$ must be mirror-symmetric around $U_{d c} / 2$.

\subsection{Analysis of Circulating Current of MMC}

The equivalent circuit of circulating current in three-phase MMC is shown in Figure 3. The output voltage of all the SMs in one arm can be seen as a controlled voltage source. $u_{x p}$, $u_{x n}, i_{x p}$ and $i_{x n}$ present the output voltage and output current of upper arm and lower arm of $x$ phase respectively $(x=\mathrm{a}, \mathrm{b}, \mathrm{c})$. $R_{x}$ denotes the loss of each arm. $i_{x}$ stands for the AC output current, and $u_{x}$ stands for $\mathrm{AC}$ output voltage. $i_{x z}$ represents the circulating current of phase $x$. $U_{d c}$ denotes the voltage of DC bus.

The circulating current is defined as:

$$
i_{x z}=\frac{i_{x n}+i_{x p}}{2}
$$

Assume that the topology of three-phase MMC is symmetric, and the AC current is allocated equally among three phases. So the relationships between circulating current and output current of upper arm and lower arm of $x$ phase can be obtained from equation (3) as formula (4). 


$$
\left\{\begin{array}{l}
i_{x p}=i_{x z}+\frac{i_{x}}{2} \\
i_{x n}=i_{x z}-\frac{i_{x}}{2}
\end{array}\right.
$$

If $m$ means the number of SMs in one arm and $C_{S M}$ denotes the capacitance in SM, then the average dynamic model of MMC can be expressed as formula (5) [19-22].

$$
\frac{d}{d t}\left[\begin{array}{l}
i_{x z} \\
u_{x p} \\
u_{x n}
\end{array}\right]=\left[\begin{array}{ccc}
-\frac{R_{r}}{L_{r}} & \frac{m}{2 L_{r}} & \frac{m}{2 L_{r}} \\
-\frac{m}{C_{S M}} & 0 & 0 \\
-\frac{m}{C_{S M}} & 0 & 0
\end{array}\right]\left[\begin{array}{l}
i_{x z} \\
u_{x p} \\
u_{x n}
\end{array}\right]+\left[\begin{array}{c}
\frac{-U_{d c}}{2 L_{r}} \\
\frac{-m_{x}}{2 C_{S M}} \\
\frac{m_{x}}{2 C_{S M}}
\end{array}\right]
$$

Applying $\mathrm{KVL}$ to $\mathrm{MMC}$ circuit, the relationship of parameters of MMC can be described by matrix (6).

$$
\frac{d}{d t}\left[\begin{array}{c}
i_{x p} \\
i_{x n}
\end{array}\right]=\left[\begin{array}{cccc}
-\frac{R_{r}}{L_{r}} & 0 & -\frac{1}{L_{r}} & 0 \\
0 & -\frac{R_{r}}{L_{r}} & 0 & -\frac{1}{L_{r}}
\end{array}\right]\left[\begin{array}{c}
i_{x p} \\
i_{x n} \\
u_{x p} \\
u_{x n}
\end{array}\right]+\left[\begin{array}{l}
\frac{U_{d c}}{2 L_{r}}-\frac{u_{x}}{L_{r}} \\
\frac{U_{d c}}{2 L_{r}}+\frac{u_{x}}{L_{r}}
\end{array}\right]
$$

Then the result of matrix (6) is showed in Equation (7).

$$
u_{x p}+u_{x n}=U_{d c}-2\left(R_{r} i_{x z}+L_{r} \frac{d i_{x z}}{d t}\right)
$$

According to Equation (7), it can be seen that the key to control circulating current is to regulate $\left(u_{x p}+u_{x n}\right)$, that is, the common-mode component of arm voltage. And Equation (7) also indicates that the imbalance between arm voltage and DC voltage is the root of circulating current.

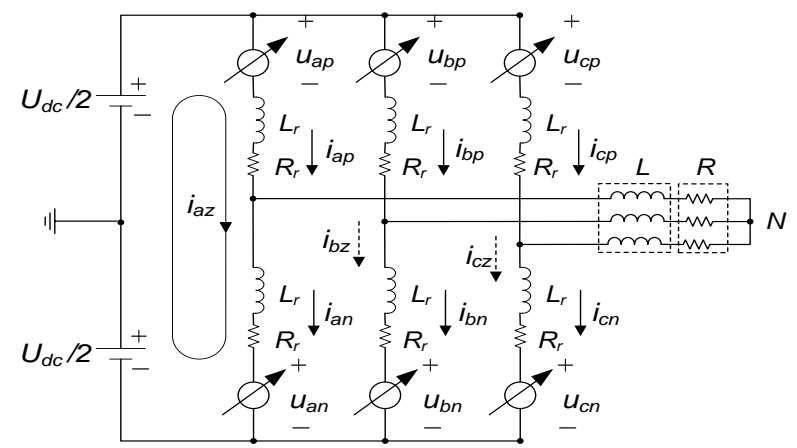

Figure 3. Equivalent circuit of circulating current in three-phase MMC

Since fundamental frequency component is contained in switching function of upper and lower arms of MMC, the capacitor voltage of SM interacts with circulating current via switching function. In this way, the low frequency harmonics in circulating current will contribute to the appearance of high frequency harmonics. Additionally, circulating current in MMC only has DC component and even frequency harmonics [14]. 


\section{Proposed Repetitive Control Scheme}

\subsection{Traditional Repetitive Control Scheme}

The ideal expression of traditional repetitive control is presented on the left of the equal sign in formula (8). And it shares a similar control performance with a group of resonant controls together with an integrate unit as presented on the right of the equal sign in Equation (8) [23].

$$
\frac{e^{-L s}}{1-e^{-L s}}=-\frac{1}{2}+\frac{\omega}{2 \pi}\left[\frac{1}{s}+\sum_{k=1}^{\infty} \frac{2 s}{s^{2}+(k \omega)^{2}}\right]
$$

Where $L$ is the period of input signal.

As $e^{-L s}$ is difficult to be realized by analog devices, repetitive control is always expressed in a discrete way as Equation (9).

$$
G_{R C}(z)=\frac{z^{-N}}{1-z^{-N}}
$$

Where $N=f_{s} / f_{o}$, and $f_{s}, f_{o}$ denote the sample frequency and fundamental frequency respectively. The sample frequency equals to the control frequency of MMC, which is $10 \mathrm{kHz}$. And fundamental frequency $f_{o}=50 \mathrm{~Hz}$.

\subsection{Proposed Repetitive Control Scheme}

The traditional repetitive control is equivalent to an integrate unit and a series of resonant controls. Therefore, by removing the integrate part of PI control, the combination of repetitive control and PI control presented in [10-13] can be simplified. And the conflict between the internal integrate unit of repetitive control and outer integrate unit in $\mathrm{PI}$ control can be avoided. The resonant controllers inside traditional repetitive controller make the system have poles with infinite gain at the points of $n \omega_{o}$. Since only even frequency harmonics exist in circulating current of MMC, the pole number is cut down and poles will only occur at the points of $2 n \omega_{o}$ in the proposed repetitive control. This improvement of repetitive control can reduce data memory and improve dynamic respond of the system [15].

The control block diagram of the proposed repetitive control is shown in Figure 4. The proportional unit is parallel with repetitive control. In this case, the $\mathrm{P}$ unit is parallel with the I unit inside repetitive control, and they realize the function of PI controller together to improve the dynamic performance of repetitive control. In addition, since the pole of traditional repetitive control is distributed on imaginary axis, the system is on the edge of oscillation [10-13]. Therefore, it is necessary to modify the traditional internal model of repetitive control. As shown in Figure $4, Q(z)$ is a constant less than 1or a low pass filter. $C(z)$ is a compensator to make up the amplitude and phase angle of control system. And $C(z)$ contains repetitive gain $\mathrm{K}_{\mathrm{RC}}$ and phase compensation link $z^{k}$. is stands for the disturbance to circulating current from fault or power sudden change, etc. $G(z)$ is the discrete function of $G(s)$, which is the control object. And the expression of $G(s)$ is $G(z)=1 /\left(2 s L_{r}+R_{r}\right)$ [11].

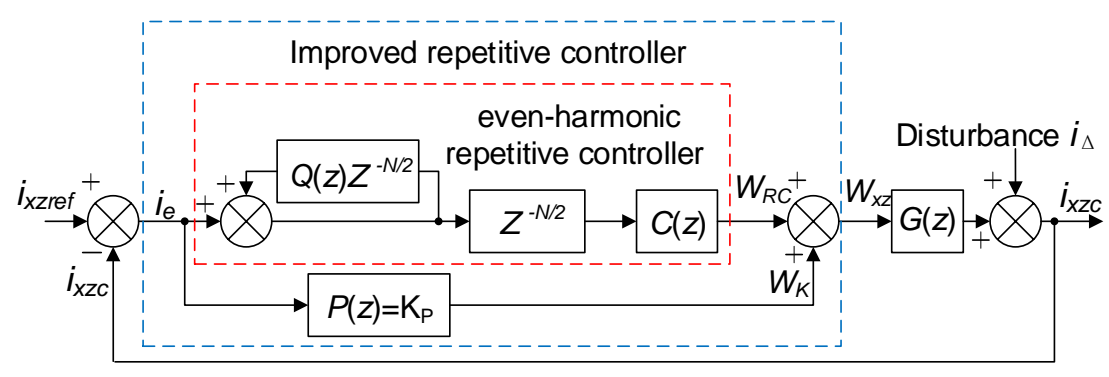

Figure 4. Control block diagram of the proposed repetitive control 


\subsection{Stability Analysis}

In Figure 4, the relationship between input $i_{e}$ and output $W_{R C}$ of the even-harmonic repetitive control is:

$$
W_{R C}(z)=\frac{C(z) \times z^{-N / 2}}{1-Q(z) \times z^{-N / 2}} \times i_{e}(z)
$$

The formula (11) shows the relationship between the input $i_{e}$ and output $W_{K}$ of proportional control $P(z)$.

$$
W_{K}(z)=\mathrm{K}_{\mathrm{P}} \times i_{e}(z)
$$

The relationships of intermediate parameters are presented in formula (12). $i_{e}$ equals to the deviation value between the input circulating current reference $i_{x z r e f}$ of phase $x$ and the actual output circulating current $i_{x z c}$. The actual output circulating current $i_{x z c}$ is equivalent to the output of the proposed improved repetitive control $W_{x z}$ multiplied by the control object $G(z)$ and then plus disturb $i_{\Delta}$. And the output of even-harmonic repetitive control $W_{R C}$ and the output of proportional control $W_{K}$ form the output of the proposed repetitive control $W_{x z}$ together.

$$
\left\{\begin{array}{l}
i_{e}(z)=i_{x z r e f}(z)-i_{x z c}(z) \\
i_{x z c}(z)=W_{x z}(z) \times G(z)+i_{\Delta}(z) \\
W_{x z}=W_{R C}(z)+W_{K}(z)
\end{array}\right.
$$

Substitute Equation (10) and Equation (11) into Equation (12), and then yields the expression of $i_{e}$ in Equation (13).

$$
i_{e}(z)=\frac{\left[i_{\text {xzref }}(z)-i_{\Delta}(z)\right] \times\left[z^{N / 2}-Q(z)\right]}{\left[\mathrm{K}_{\mathrm{P}} G(z)+1\right] \times\left[z^{N / 2}-Q(z)+\frac{C(z) G(z)}{\mathrm{K}_{\mathrm{P}} G(z)+1}\right]}
$$

Due to Equation (13), the characteristic equation of system is as follow.

$$
\left[\mathrm{K}_{\mathrm{P}} G(z)+1\right] \times\left[z^{N / 2}-Q(z)+\frac{C(z) G(z)}{\mathrm{K}_{\mathrm{P}} G(z)+1}\right]=0
$$

And this characteristic equation contains following two parts:

$$
\begin{aligned}
& F_{1}(z)=\mathrm{K}_{\mathrm{P}} G(z)+1=0 \\
& F_{2}(z) z^{N / 2}-Q(z)+\frac{C(z) G(z)}{\mathrm{K}_{\mathrm{P}} G(z)+1}=0
\end{aligned}
$$

According to the stability theory of discrete control system, the necessary and sufficient condition of a stable system is that all the roots of the characteristic equation are situated within the unit circle. So the characteristic roots of $F_{1}(z)$ and $F_{2}(z)$ in formula (15) are all required to be within unit circle respectively to maintain the system stability. $F_{1}(z)$ represents the stability condition of proportional control and $F_{2}(z)$ stands for that of the even-harmonic repetitive control, and the corresponding control objects are $G(z)$ and $G(z) /\left(K_{P} G(z)+1\right)$. As the proportional control is stable [24], so the key point of the system stability condition is that characteristic roots of $F_{2}(z)$ need to satisfy formula (16). 


$$
\left|z^{N / 2}\right|=\left|Q(z)-\frac{C(z) G(z)}{\mathrm{K}_{\mathrm{P}} G(z)+1}\right|<1
$$

Where $z=e^{j \omega T}, 0<\omega<\pi / T$.

\subsection{Design of Proposed Repetitive Controller}

In order to keep the stability of the circulating current suppressing system, the design of $C(z)$ and $Q(z)$ needs to satisfy formula (16).

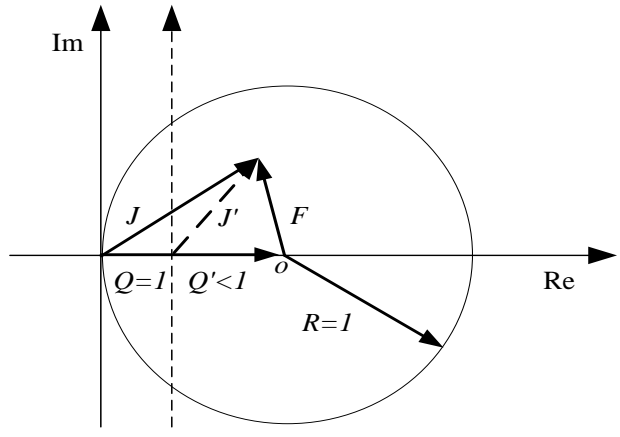

Figure 5. Diagram of stability of the proposed repetitive control

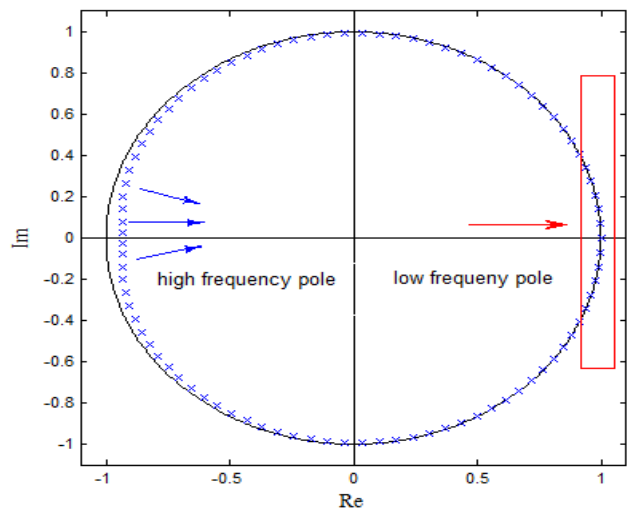

Figure 6. Pole diagram of closed loop characteristic equation of the control system

Formula (16) are represented by vectors in Figure 5. The vectors are $J(z), F(z)$ and $\mathrm{Q}(\mathrm{z})$, where $J(z)=C(z) G(z) /\left(\mathrm{K}_{\mathrm{P}} G(z)+1\right)$ and $F(z)=Q(z)-J(z)$. Furthermore, the radius $\mathrm{R}$ of the unit circle is 1 and the magnitude of $F(z)$ is required to be less than 1. In this figure, $Q(z)$ presents the contribution to system robustness in medium and high frequency band. In low and medium frequency band, the error is not big because of $C(z)$ which compensates the phase and amplitude of the control system, so $J(z)$ nearly coincides with the real axis, and $Q(z)$ is not necessary in this case. However, in medium and high frequency band, the error is inevitable and the effect of compensation is unwarranted. If $Q(z)$ is removed or $Q(z)=1$, then the center of the unit circle is at point $(1,0)$ and the circle is tangent to the imaginary axis with only first and fourth quartile included in unit circle. In this situation, if the phase of $J(z)$ exceeds $90^{\circ}$, then its end must be out of the unit circle, which implies the instability of the system because $F(z)>1$. Therefore, in medium and high frequency band, $Q(z)<1$ is necessary and it is equivalent to a right shift of imaginary axis as showed in Figure 6 with imaginary line, then a part of the second quartile and third quartile is also included in unit circle. In this case, the end of $J(z)$ can be kept within the circle even if the phase of $J(z)$ exceeds $90^{\circ}$, which means the system is still stable since $F(z)<1$.

Nevertheless, sufficient gain is also needed to realize harmonic compensation and nosteady-state error track, especially in low-medium frequency band. So in this way, the $Q(z)$ is better to be a low pass filter rather than a constant. Then it can satisfy both requirements of low frequency band and high frequency band. Then, if a low pass filter is adapted, for one thing, the center of the unit circle is near point $(1,0)$ in low-medium frequency band, which guarantees an adequate gain. And for another, the imaginary axis will shift right a certain distance in mediumhigh frequency band, which improves the stability of the system. To conclude, the adaptation of the low pass filter is the optimization of stability and static error.

Table 1 shows the parameters of the proposed repetitive control. According to Juli stability criterion and the transfer function of control object $G(z)$, the value range of $K_{p}$ is positive and less than 30, and after comparing amplitude-frequency characteristic performances of different $K_{p}$ value, $K_{p}$ is set as 10 in this paper [24]. As mentioned above, $J(z)$ is with no phase shift and no gain in low-medium frequency band, so the part without $\mathrm{K}_{\mathrm{RC}}$ of $J(z)$ can be regarded as 1 , and then $\mathrm{J}(\mathrm{z})$ is equivalent to $\mathrm{K}_{\mathrm{RC}}$ [24]. 
Table 1. The Parameters of the Proposed Repetitive Controller

\begin{tabular}{cc}
\hline Parameters & Value \\
\hline Proportional controller $\mathrm{K}_{\mathrm{P}}$ & 10 \\
Gain of repetitive controller $\mathrm{K}_{\mathrm{RC}}$ & 0.15 \\
Internal model filter $\mathrm{Q}(\mathrm{z})$ & $Q(z)=\frac{z^{-1}+2+z}{4}$ \\
& $\mathrm{Z}^{3}$ \\
Phase compensation link & $10 \mathrm{kHz}$ \\
Carrier frequency & $1200 \mathrm{~Hz}$ \\
System bandwidth &
\end{tabular}

Then formula (16) can be rewritten as follow:

$$
\left|Q(z)-\mathrm{K}_{\mathrm{RC}}\right|<1
$$

$\mathrm{K}_{\mathrm{RC}}$ must be positive because if the direction of $J(z)$ is opposite and then the end of it would be out of the unit circle, so the value range of $\mathrm{K}_{\mathrm{RC}}$ can be obtained as formula (18).

$$
0<\mathrm{K}_{\mathrm{RC}}<|Q(z)|+1
$$

The $Q(z)$ is a low pass filter with the value always less than 1 , and it becomes smaller as the frequency increases. After overall consideration, $K_{R C}$ is 0.15 and the phase compensation link is $z^{3}$ in this paper.

Figure 6 shows the pole diagram of closed loop characteristic equation of the control system with designed parameters. In Figure 6, the low frequency poles (such as second, fourth and sixth frequency harmonics) are extremely close to the edge of the unit circle, but they are still inside the circle. And this feature warrants sufficient gain at these low frequency points and would contribute to higher respond speed and better tracking property. As for poles of high frequency, they are polarized inward (as the blue arrow shows) and ensure the steadiness of the system. Therefore, it can be seen that the circulating current suppression under the proposed repetitive control fully satiates the conditions of system stability.

\section{Simulation Realization}

\subsection{Simulation Model and Parameters}

A three-terminal MMC system with 129-level is built in PSCAD/EMTDC, and the topology of each terminal is the same. Sending end 1 and 2 adapt constant active power control and constant reactive control, and receiving end 3 takes constant DC voltage control and constant reactive power control. Traditional PI control based on dq decoupling method and proposed repetitive control are employed to each terminal at different time to restrain circulating current. The system is in unit power factor operation and uses nearest level modulation (NLM) [25-27]. The related simulation parameters are listed in Table 2.

Table 2. Simulation parameters

\begin{tabular}{cccc}
\hline Model parameters & Value & Model parameters & Value \\
\hline Line voltage of power grid $/ \mathrm{kV}$ & 110 & Capacitance of SM $/ \mathrm{mF}$ & 6.9 \\
Equivalent resistor of power grid $/ \Omega$ & 1.8 & SM switching frequency $/ \mathrm{Hz}$ & 216 \\
Equivalent inductance of power grid $/ \mathrm{mH}$ & 6 & System rated frequency $/ \mathrm{Hz}$ & 50 \\
Ratio of transformer & $110 / 168$ & Rated direct voltage $/ \mathrm{kV}$ & 320 \\
Carrier frequency $/ \mathrm{Hz}$ & 10000 & SM rated capacitor voltage $/ \mathrm{kV}$ & 2.5 \\
Voltage modulation degree of rectifier side & 0.845 & Voltage modulation degree of inverter side & 0.888 \\
\hline
\end{tabular}

\subsection{Simulation Result}

The simulations are set in four different conditions, namely the normal operation, single phase to ground fault happens on the AC side of end 3 , line fault happens at a DC line and three-phase short circuit fault happens at end 3 . In all conditions, the system initially operates 
without any circulating current control strategy, and then traditional PI control based on dq decoupling method is applied to repress circulating current during $1.5 \mathrm{~s}$ to $2 \mathrm{~s}$, and the proposed repetitive control replaces $\mathrm{PI}$ control after $2 \mathrm{~s}$.

\subsubsection{Condition 1: Normal Operation}

The condition 1 is that the system operates in normal condition. The Figure 7 shows the simulation results of three-phase circulating current of end 3 in normal operation. The Figure $7(\mathrm{a})$ is the result of the circulating current suppression method based on traditional $\mathrm{PI}$ control, and Figure $7(\mathrm{~b})$ is the result of that based on the proposed repetitive control. It is clear that the proposed repetitive control presents superior circulating current restraining ability than $\mathrm{PI}$ control, and the harmonics in circulating current is nearly eliminated after the proposed strategy is used.

The Figure 8 shows the capacitor voltage of a sub-module of end 3 in normal operation. It can be seen from Figure 8 that the peak-valley difference of SM voltage is suppressed to be approximately $0.2 \mathrm{kV}$ after the PI control is carried out at $1.5 \mathrm{~s}$, and the fluctuation of SM voltage is further restrained after the proposed method is applied at $2 \mathrm{~s}$.

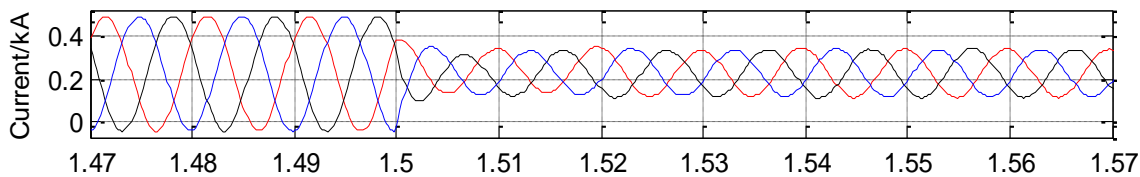

(a) Circulating current when PI control applied at $1.5 \mathrm{~s}$

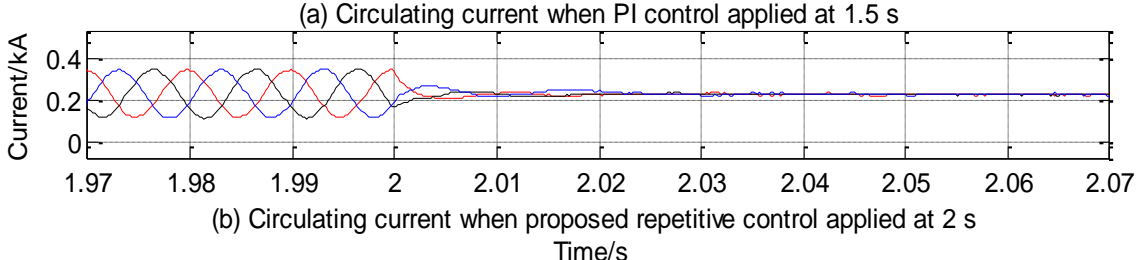

Figure 7. Three-phase circulating current of end 3 in condition 1
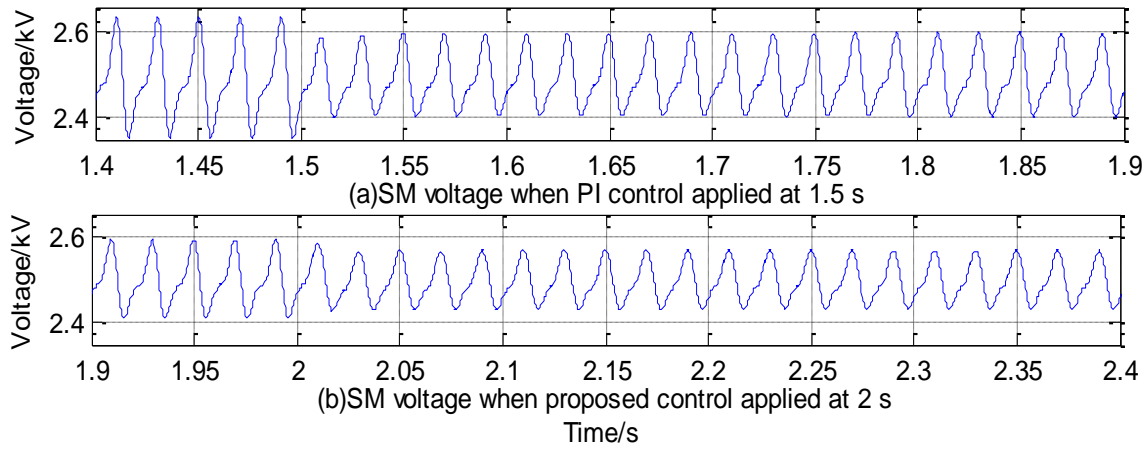

Figure 8. SM capacitor voltage of end 3 in condition 1

\subsubsection{Condition 2: One phase to ground fault happens at the $A C$ side of end 3}

The condition 2 is that the end 3 has one phase earth fault lasts for $0.1 \mathrm{~s}$ at $1.6 \mathrm{~s}$ and $2.2 \mathrm{~s}$ at the $\mathrm{AC}$ side of it.

The Figure 9 and Figure 10 are the simulation results of three-phase circulating current and SM capacitor voltage of end 3 in condition 2 respectively. As shown in Figure 9, both the traditional $\mathrm{PI}$ control and the proposed repetitive control are able to restore to original condition after the one phase fault is removed, but the two non-fault phases of the latter has the maximum offset less than $0.1 \mathrm{kA}$ from normal position while that of the former is more than $0.2 \mathrm{kA}$. And the fluctuation of circulating current is wiped off. 
And in Figure 10, while applying repetitive control, the fluctuation amplitude of SM voltage is smaller than that when PI control is employed during the fault. And after the fault is removed, the amplitude of SM voltage is restored to original condition when proposed repetitive control is applied.
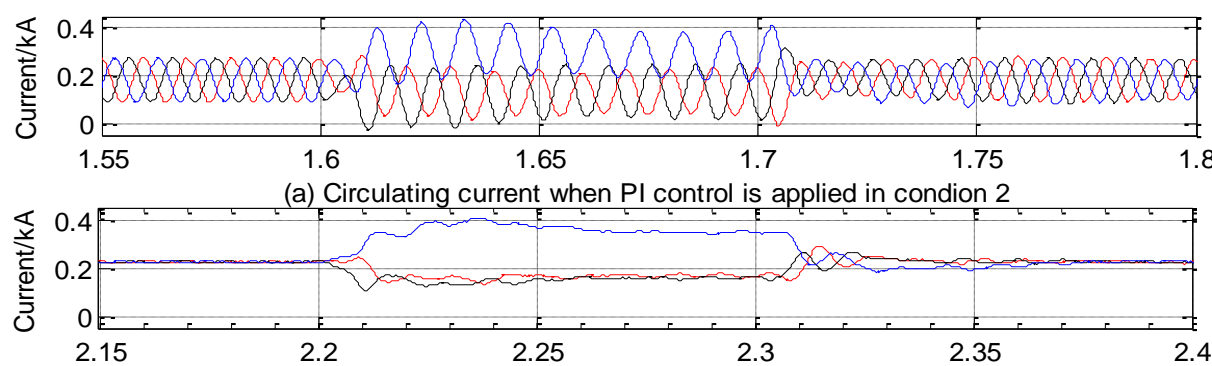

(b) Circulating current when proposed control is applied in condition 2 Time/s

Figure 9. Three-phase circulating current of end 3 in condition 2
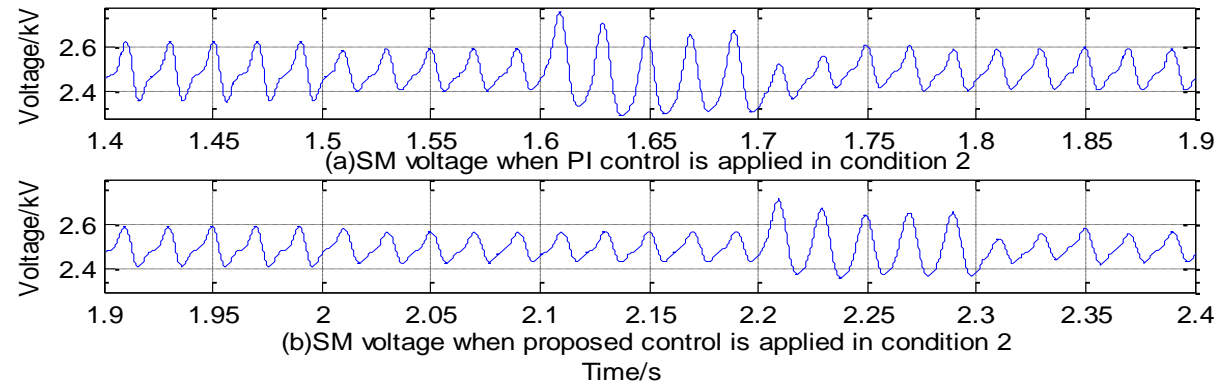

Figure 10. SM capacitor voltage of end 3 in condition 2

\subsubsection{Condition 3: line fault happens in DC line}

The condition 3 is that earth fault occurs at a DC line for $1 \mathrm{~s}$ at $1.6 \mathrm{~s}$ and $2.2 \mathrm{~s}$. The Figure $11(\mathrm{a})$ and (b) present the simulation results of three-phase circulating current of end 3. And the simulation results of SM voltage are showed in Figure 12(a) and (b). In all figures, the fluctuations result from DC line fault are cleared up in $0.1 \mathrm{~s}$, but repetitive control shows better anti-interference capability than $\mathrm{PI}$ control with smaller maximum amplitude of circulating current and SM voltage in fault period.
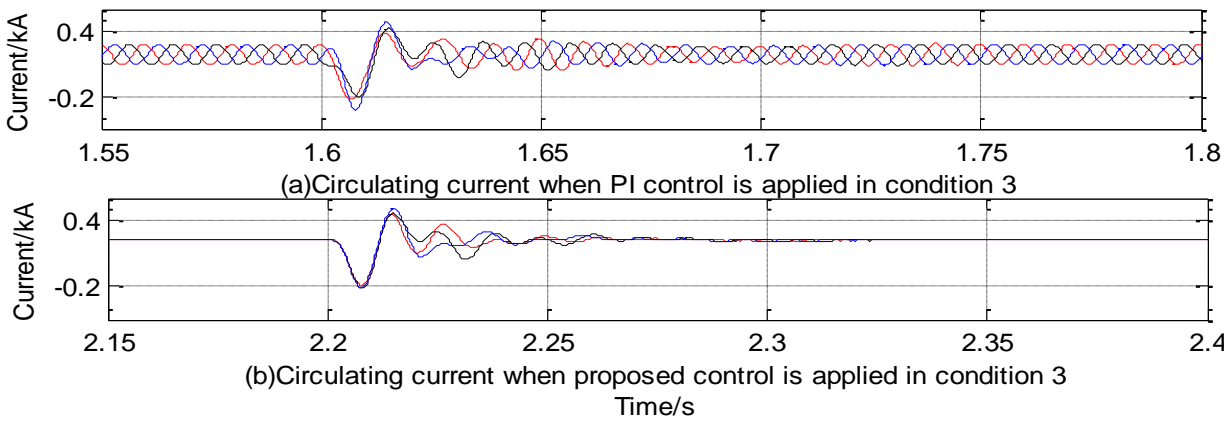

Figure 11. Three- phase circulating current of end 3 in condition 3 


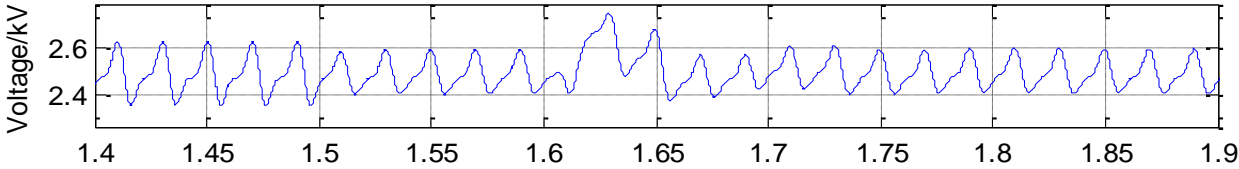

(a) SM voltage when PI control is applied in condition 3

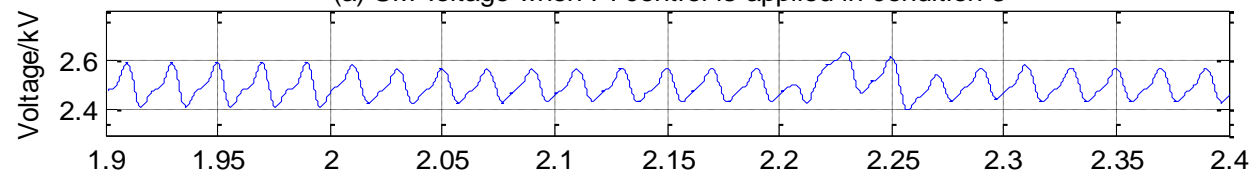

(b) SM voltage when proposed control is applied in condition 3

Time/s

Figure 12. SM capacitor voltage of end 3 in condition 3

\subsubsection{Condition 4: Three-phase short circuit fault happens at the $A C$ side of end 3}

The condition 4 is that three-phase short circuit fault happens at the AC side end 3 for $0.001 \mathrm{~s}$ at $1.6 \mathrm{~s}$ and $2.2 \mathrm{~s}$.

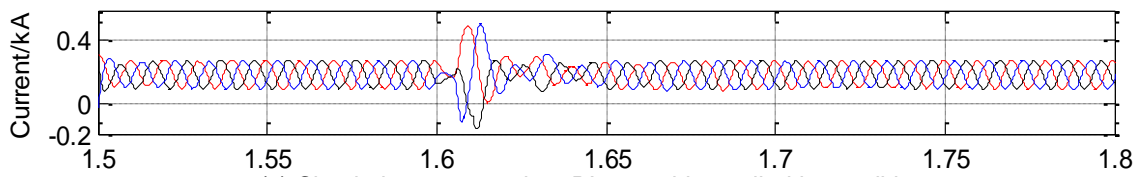

(a) Circulating current when PI control is applied in condition 4

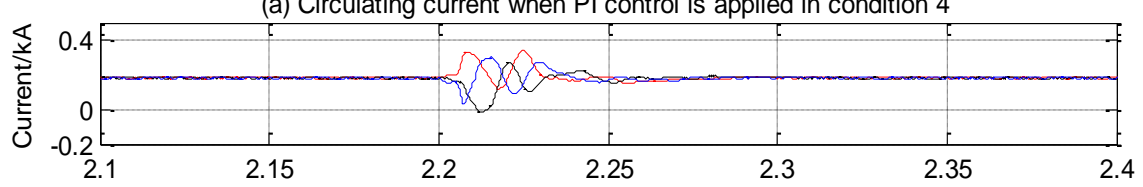

(b) Circulating current when proposed control is applied in condition 4 Time/s

Figure 13. Three-phase circulating current of end 3 in condition 4
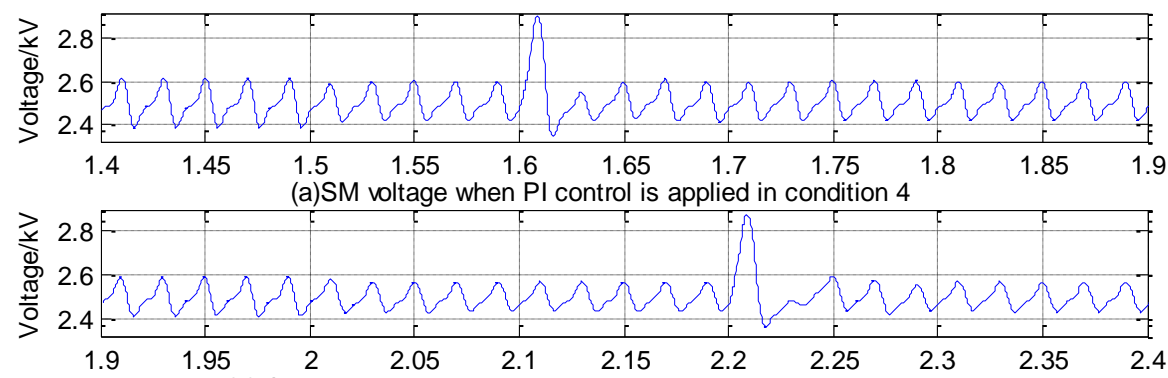

(b) Circulating current when proposed control is applied in condition 4

Time/s

Figure 14. SM capacitor voltage of end 3 in condition 4

Figure 13 and Figure 14 present the simulation results of three-phase circulating current and SM voltage of end 3 . It can be seen that proposed repetitive control shows similar superiority of anti-interference capability with the above conditions.

\section{Conclusion}

In this paper, the structure and operating principle of MMC-MTDC and the circulating current mechanism of MMC are presented firstly, then the characteristic of traditional repetitive control is analyzed. Afterwards, by considering even-harmonic characteristic of MMC circulating 
current and equivalent embedded integration structure of repetitive control, an improved repetitive control strategy is proposed. The pole analysis verified the stability of proposed controller with designed parameters. Finally, the simulation verifications are based on the threeterminal 129-level MMC mode in PSCAD/EMTDC. The effectiveness of the proposed control is proved by comparing with traditional $\mathrm{PI}$ control based on dq decoupling method in both balance grid condition and unbalance grid condition. The four different conditions of simulation are normal operation condition, one phase earth fault condition, line fault in dc-line condition and three-phase short circuit fault condition. The simulation results show better circulating current suppression ability and anti-interference capability of the proposed repetitive control scheme than PI control in all conditions studied above. And the results also indicate that the proposed repetitive controller can suppress the fluctuation of SM voltage more effectively than PI control.

\section{Acknowledgements}

This work is supported by the National Natural Science Foundation of China (51520105011).

\section{References}

[1] Wang P, Li Z, Zhang X P. Start-Up Sequences of an Offshore Integrated MMC MTDC System. IET International Conference on AC and DC Power Transmission. Birmingham. 2015: 1-7.

[2] Livermore L, Liang J, Ekanayake J. MTDC VSC Technology and its applications for wind power. Universities Power Engineering Conference. Cardiff. 2010: 1-6.

[3] Kouro S, Malinowski M, Gopakumar K. Recent Advances and Industrial Applications of Multilevel Converters. IEEE Transactions on Industrial Electronics. 2010; 57(8): 2553-2580.

[4] Madichetty S, Dasgupta A. Modular Multilevel Converters Part-I: A Review on Topologies, Modulation, Modeling and Control Schemes. International Journal of Power Electronics \& Drive Systems. 2014; 4(1): 36-50.

[5] Madichetty S, Abhijit D, Jinka S. A Survey and experimental verification of Modular Multilevel Converters. International Journal of Power Electronics \& Drive Systems. 2014; 4(3): 363-375.

[6] Tu Q, Xu Z, Xu L. Reduced Switching-Frequency Modulation and Circulating Current Suppression for Modular Multilevel Converters. IEEE Transactions on Power Delivery. 2012; 26(3): 2009-2017.

[7] Bahrani B, Debnath S, Saeedifard M. Circulating Current Suppression of the Modular Multilevel Converter in a Double-Frequency Rotating Reference Frame. IEEE Transactions on Power Electronics. 2016; 31(1): 783-792.

[8] Li Z, Wang P, Chu Z. An Inner Current Suppressing Method for Modular Multilevel Converters. IEEE Transactions on Power Electronics. 2013; 28(11): 4873-4879.

[9] Li Y, Wang F. Arm inductance selection principle for modular multilevel converters with circulating current suppressing control. IEEE Applied Power Electronics Conference and Exposition. Long Beach. 2013: 1321-1325.

[10] Zhang M, Huang L, Yao W. Circulating Harmonic Current Elimination of a CPS-PWM-Based Modular Multilevel Converter With a Plug-In Repetitive Controller. IEEE Transactions on Power Electronics. 2014; 29(4): 2083-2097.

[11] He L, Zhang K, Xiong J. A Repetitive Control Scheme for Harmonic Suppression of Circulating Current in Modular Multilevel Converters. IEEE Transactions on Power Electronics. 2015; 30(1): 471481.

[12] Madichetty S, Dasgupta A, Mishra S. Application of an Advanced Repetitive Controller to Mitigate Harmonics in MMC with APOD Scheme. IEEE Transactions on Power Electronics. 2016; 31(9): $6112-6121$

[13] Moranchel M, Sanz I, Bueno E J. Circulating current elimination in Modular Multilevel Converter with repetitive controllers. IECON 2016-42nd Annual Conference of the IEEE Industrial Electronics Society. Florence. 2016: 6476-6481.

[14] Ilves K, Antonopoulos A, Norrga S. Steady-State Analysis of Interaction between Harmonic Components of Arm and Line Quantities of Modular Multilevel Converters. IEEE Transactions on Power Electronics. 2012; 27(1): 57-68.

[15] Yang S, Wang P, Tang Y. Even-harmonic repetitive control for circulating current suppression in Modular Multilevel Converters. IEEE Applied Power Electronics Conference and Exposition. Long Beach. 2016: 3591-3597.

[16] Wang J, Liang J, Wang C. Circulating current suppression for MMC-HVDC under unbalanced grid conditions. IEEE Industry Applications Society Meeting. Portland. 2016: 1-9. 
[17] Moon JW, Park JW, Kang DW. A Control Method of HVDC-Modular Multilevel Converter Based on Arm Current under the Unbalanced Voltage Condition. IEEE Transactions on Power Delivery. 2015; 30(2): 529-536.

[18] Zhao $\mathrm{Y}, \mathrm{Hu} \mathrm{XH}$, Tang G F. A study on MMC model and its current control strategies. IEEE International Symposium on Power Electronics for Distributed Generation Systems. Hefei. 2010: 259264.

[19] Jovcic D, Jamshidi Far A. Phasor Model of Modular Multilevel Converter with Circulating Current Suppression Control. IEEE Transactions on Power Delivery. 2015; 30(4): 1889-1897.

[20] Antonopoulos A, Angquist L, Nee HP. On dynamics and voltage control of the Modular Multilevel Converter. European Conference on Power Electronics and Applications. Barcelona. 2009: 1-10.

[21] Peralta J, Saad H, Dennetiere S. Detailed and averaged models for a 401-level MMC-HVDC system. Power and Energy Society General Meeting. Vancouver. 2013: 1.

[22] Saad H, Peralta J, Dennetière S. Dynamic Averaged and Simplified Models for MMC-Based HVDC Transmission Systems. IEEE Transactions on Power Delivery. 2013; 28(3): 1723-1730.

[23] $\mathrm{Ni} \mathrm{J.} \mathrm{Research} \mathrm{on} \mathrm{repetitive} \mathrm{control} \mathrm{in} \mathrm{active} \mathrm{power} \mathrm{filter.} \mathrm{Master} \mathrm{Thesis.} \mathrm{Hangzhou:} \mathrm{Zhejiang}$ University; 2013.

[24] Zhang Y. Research on single phase unified power quality controller. PhD Thesis. Wuhan: Huazhong University of Science and Technology; 2009.

[25] Xu J, Zhao C, Liu W. Accelerated Model of Modular Multilevel Converters in PSCAD/EMTDC. IEEE Transactions on Power Delivery. 2013; 28(1): 129-136.

[26] Guan M, Xu Z, Tu Q. Nearest level modulation for modular multilevel converters in HVDC transmission. Automation of Electric Power Systems. 2010; 34(2): 48-52.

[27] Deng Y, Saeedifard M, Harley R G. An improved nearest-level modulation method for the modular multilevel converter. IEEE Applied Power Electronics Conference and Exposition. Charlotte. 2015: $1595-1600$ 